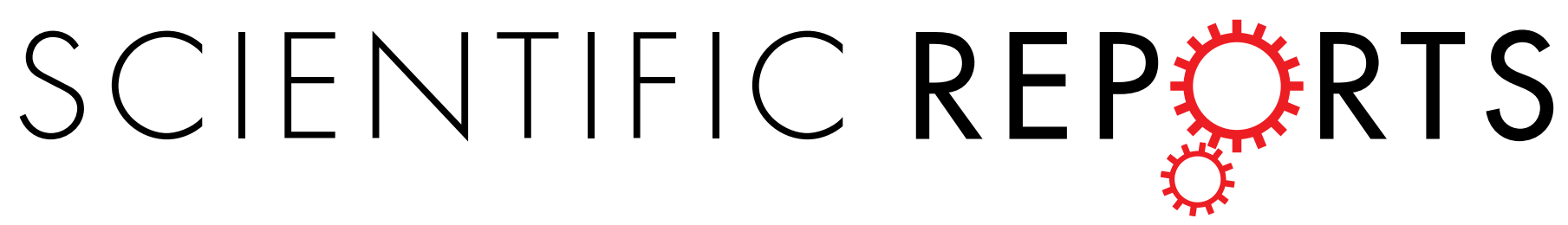

\title{
Erratum: Modulation of $\lg \beta$ is essential for the $B$ cell selection in germinal center
}

Kagefumi Todo, Orie Koga, Miwako Nishikawa \& Masaki Hikida

Scientific Reports 5:10303; doi: 10.1038/srep10303; published online 18 May 2015; updated on 05 August 2015

The Competing financial interests statement in this Article should read:

"This work was supported in part by Astellas Pharma Inc. in the Formation of Innovation Center for Fusion of Advanced Technologies Program." 